\title{
Management of Hepatocellular Carcinoma: Updated Review
}

\author{
Tamer Elbaz ${ }^{1}$, Mohamed El Kassas ${ }^{2}$, Gamal Esmat ${ }^{1}$ \\ ${ }^{1}$ Endemic Medicine and Hepatogastroenterology Department, Faculty of Medicine, Cairo University, Cairo, Egypt; ${ }^{2}$ Hepatology \\ Department, National Hepatology and Tropical Medicine Research Institute, Cairo, Egypt. \\ Email: m_elkassas@yahoo.com
}

Received December $12^{\text {th }}, 2012$; revised January $14^{\text {th }}, 2013$; accepted January $22^{\text {nd }}, 2013$

Copyright (C) 2013 Tamer Elbaz et al. This is an open access article distributed under the Creative Commons Attribution License, which permits unrestricted use, distribution, and reproduction in any medium, provided the original work is properly cited.

\begin{abstract}
Hepatocellular carcinoma (HCC) represents one of the most challenging potentially curable tumors with high incidence, prevalence and mortality rates. For proper assessment, prognosis estimation and treatment decisions, at least seven important guidelines and staging systems were designated. Proper treatment needs the interaction of multidisciplinary HCC clinic to choose the most appropriate line of treatment. The different modalities of management include resection (surgery or transplantation), local ablation, chemoembolization, radioembolization and molecular targeted therapies with a wide range of investigational drugs that developed after the FDA approved sorafenib. Downstaging and bridging are two important strategies to manage HCC patients who will undergo liver transplantation to improve their postoperative survival. Finally, survival and prognosis depends on several prognostic factors that are either patient related or tumor related. In our study, we aim to provide an updated comprehensive review of the different aspects of liver cancer management starting from staging systems to the different applied treatment modalities.
\end{abstract}

Keywords: Hepatocellular Carcinoma; Staging; Molecular Targeted Therapies

\section{Introduction}

Hepatocellular Carcinoma (HCC) is the sixth most common cancer worldwide, accounting for $7 \%$ of all cancers and an estimated incidence of 749,000 new cases every year. It is considered to be the third cause of cancer related deaths $(692,000$ cases $)$. The highest incidence rates of HCC (around $85 \%$ of cases) are present in East Asia, sub-Saharan Africa, and Melanesia [1]. Management of HCC starts by identifying its stage and underlying liver status then choosing the most appropriate line of therapy (surgical, locoregional, radiological and medical) aiming to improve the survival and/or the quality of life of the patient. In that context, this review will provide an updated status of the different aspects of liver cancer management from the different staging systems to the applied and future treatment modalities.

\section{Staging}

Clinical staging systems are primarily made to assure well designated guidelines for assessment, prognosis estimation and treatment decisions. For the time being, at least seven different staging systems are present for management of HCC. Each one of these staging systems has its advantages as well as some related defects [2]. We will review briefly these guidelines with special emphasis on their characteristics.

\subsection{TNM Classification}

The TNM (tumor size-lymph nodes-metastasis) staging was established through a multicenter international study for management of patients with HCC [3]. This classification depends on tumor extension, microvascular invasion, and liver fibrosis. Its main role relies on using it as a surgical staging system while it has no role in non surgical patients [4].

\subsection{Okuda Staging System}

It is the first known staging system for HCC, with a wide use in Japan and Eastern countries since its publication in 1985. It depends on factors related to tumor extension (more or less than $50 \%$ of liver involved) and to liver functions [5]. Okuda staging system is much suitable for patients with advanced HCC and who are not usually fit for any intervention maneuvers. Recent advances in di- 
agnostic modalities created a large group of patients with small lesions which are not well dealt with this staging system. Another drawback for this system is the lack of prognostic indicators.

\subsection{CLIP (Cancer of Liver Italian Program) Staging System}

The CLIP score has been established after a retrospective Italian study that included new prognostic factors, like alpha-fetoprotein and portal vein thrombosis [6]. Many following studies validated this system and proved its superiority and accuracy compared to systems like Okuda or TNM staging systems [7], with specific advantage as a prognostic module even more than some recent and widely accepted systems like Barcelona Clinic Liver Cancer (BCLC) and the Japan Integrated Scoring (JIS) system [8]. The most important criticism is the weak differentiation in early tumor stages, the difficult application for treatment strategy based on this system beside the poor efficacy in terms of survival [9].

\subsection{GRETCH (Group d'Etude et De Traitement du Carcinome Hepatocellulaire) Score}

This French staging system developed from results of a multicenter international study that included the performance status as one of its parameters [10]. This system is not widely used and was not well validated due to presence of some weak points like the poor differentiation of tumor spread.

\subsection{CUPI (Chinese University Prognostic Index) System}

The CUPI system was performed in Hong Kong after a study on Chinese patients who suffered from HCC [11]. The score system depends on variables such as tumor extension (with TNM classification), liver profile and presence of symptoms at presentation. The main drawback is its suitability for advanced rather than early cases and its dependence on a study population with a predominant HBV background [12].

\subsection{JIS (Japanese Integrated Staging) Score}

The JIS score is a simple and easy used staging system which combines the Child-Pugh grade with the TNM classification with some modifications introduced by Liver Cancer Study Group of Japan [13]. For the time being, this score is used only by the local Japanese guidelines [14].

\subsection{BCLC Staging System}

This staging system is one of the most accepted and widely used systems. It was developed after a retrospec- tive analysis of several cohort studies for several HCC stages [15]. This system depends on classification of HCC into five major categories: very early, early, intermediate, advanced, and terminal stages. For each stage, many prognostic variables are considered, life expectancy is estimated and the most suitable management plan is suggested. The system includes variables related to tumor specifications, liver profile, and performance status of the patient. BCLC is the most commonly used staging system in Europe and it has been approved by the European Association for the Study of the Liver (EASL) and the American Association for the Study of Liver Diseases (AASLD) [16]. The most commonly used staging systems and the main characteristics, uses and comments on each one are listed in Table 1.

\section{Treatment}

HCC recently appeared as a potentially curable disease with many treatment options that depend on patient factors as the performance status and operability, and tumor related factors like size, location and the presence or absence of extratumoral spread. Some of the previously discussed staging systems offer a guide for treatment plans. The most commonly used treatment modalities will be discussed.

Table 1. Most commonly used staging systems.

\begin{tabular}{|c|c|c|}
\hline $\begin{array}{l}\text { Staging } \\
\text { system }\end{array}$ & $\begin{array}{l}\text { Characteristic } \\
\text { determinants }\end{array}$ & $\begin{array}{l}\text { Usages, advantages } \\
\text { and comments }\end{array}$ \\
\hline TNM & $\begin{array}{c}\text { Tumor extension } \\
\text { Microvascular invasion } \\
\text { Liver fibrosis }\end{array}$ & $\begin{array}{l}\text { A surgical staging } \\
\text { system }\end{array}$ \\
\hline Okuda & $\begin{array}{c}\text { Tumor extension (more } \\
\text { or less than } 50 \% \text { of liver } \\
\text { involved) } \\
\text { Liver functions }\end{array}$ & $\begin{array}{c}\text { Mainly for patients with } \\
\text { advanced HCC }\end{array}$ \\
\hline CLIP & $\begin{array}{l}\text { Alpha-fetoprotein } \\
\text { Portal vein thrombosis }\end{array}$ & More prognostic module \\
\hline GRETCH & $\begin{array}{c}\text { Inclusion of } \\
\text { performance status }\end{array}$ & $\begin{array}{l}\text { Poor differentiation of } \\
\text { tumor spread }\end{array}$ \\
\hline CUPI & $\begin{array}{l}\text { Tumor extension (with } \\
\text { TNM classification) } \\
\text { Liver profile Patients } \\
\text { symptoms }\end{array}$ & $\begin{array}{l}\text { Suitable more for } \\
\text { advanced rather } \\
\text { than early cases }\end{array}$ \\
\hline JIS & $\begin{array}{l}\text { Combines the Child-Pugh } \\
\text { grade with the TNM } \\
\text { classification }\end{array}$ & $\begin{array}{l}\text { Simple and easy used } \\
\text { Widely used in Japan }\end{array}$ \\
\hline BCLC & $\begin{array}{l}\text { Tumor specifications } \\
\text { Liver profile } \\
\text { Performance status }\end{array}$ & $\begin{array}{l}\text { Classifies HCC into } \\
\text { five major categories: } \\
\text { very early, early, } \\
\text { intermediate, advanced, } \\
\text { and terminal stages. } \\
\text { Prognosis, life } \\
\text { expectancy and the } \\
\text { suitable management } \\
\text { plan is considered for } \\
\text { each category }\end{array}$ \\
\hline
\end{tabular}




\subsection{Multidisciplinary Management}

Notably, there is wide heterogeneity in HCC presentation, patient variations as candidates for recommended treatments, and increasingly complex available therapeutic options with diverse responses to these therapies in clinical practice [17]. HCC is also known by its highly variable biologic behavior and the frequent coexistence of chronic liver disease and cirrhosis with HCC in affected patients [18]. In context of these reasons, it is important to manage HCC patients by multidisciplinary HCC teams. The multidisciplinary clinic includes heaptologists, medical and surgical oncologists, transplantation surgeons, diagnostic and interventional radiologists, pathologists, nurses and nurse practitioners [19].

\subsection{Resection}

Surgery is the cornerstone of HCC management. Resection and transplantation represent the best treatment options for patients who are candidates for such treatment modality in terms of survival [20].

\subsection{Liver Transplantation}

Liver transplantation is the first treatment choice for patients with small multinodular tumors ( $\leq 3$ nodules $\leq 3$ $\mathrm{cm}$ ) or those with single tumors $\leq 5 \mathrm{~cm}$ and advanced liver dysfunction.

One of the most famous guidelines for transplantation in HCC patients is the Milan criteria applied for patients with a single $\mathrm{HCC} \leq 5 \mathrm{~cm}$ or multinodular lesions with a maximum of three nodules $\leq 3 \mathrm{~cm}$ each [21]. Two main approaches were followed in many trials; the first approach was to study the extension of transplantation legibility criteria to cover more patients in terms of tumor size and number. The most validated trial was the expansion to UCSF criteria (single nodule $\leq 6.5 \mathrm{~cm}$ or 2 - 3 nodules $\leq 4.5 \mathrm{~cm}$ and total tumor diameter $\leq 8 \mathrm{~cm}$ ) which involves around $5 \%-10 \%$ of all transplant enlisted patients [22]. The second approach was the attempts for down staging of cases with much advanced HCC for adaption to Milan criteria before transplantation [23].

\subsection{Local Ablation}

Local ablation techniques have been developed for patients who are not surgically fit. They are performed either through percutaneous approach or less commonly by laparoscopic introduction. These maneuvers include percutaneous ethanol injection (PEI), percutaneous acetic acid injection, radiofrequency ablation (RFA), microwave ablation, cryoablation, laser-induced thermotherapy, high-intensity focused ultrasound, and irreversible electroporation [24].
The first used technique was PEI, which induces coagulative necrosis of the lesion as a result of cellular dehydration, protein denaturation, and chemical occlusion of small tumor vessels as an effect of the injected absolute alcohol. The use of temperature in ablation techniques appeared later, with either using the heating techniques like radiofrequency ablation (RFA), microwave ablation, and laser ablation or through using cryoablation which causes direct tumor freezing [25]. The estimated 5 year survival in patients with early $\mathrm{HCC}$ was $47 \%-53 \%$ [26]. RFA is the most commonly used local ablative technique and it has largely replaced PEI with proven superiority regarding the survival and recurrence in fewer treatment sessions [27]. It depends on energy production that induces coagulative necrosis of the tumor with safety margins of the apparently healthy tissue around the lesion.

\subsection{Chemoembolization}

Transarterial chemoembolization (TACE) is considered as the most commonly used initial treatment for unresectable HCC [28]. The well characterized angiogenic activity of HCC was the rationale behind TACE use. This technique depends on the intra-arterial infusion of a cytotoxic chemotherapeutic agent emulsioned with Lipiodol followed by embolization of the feeding vessels through a trans-arterial catheter [29].

The maximum and sustained retention of the chemical agent is used as a measure for the success of TACE. This allows the use of embolic microspheres which have the ability to sequester chemotherapeutic agents and release them in a controlled manner over a one week period and a subsequent increase of the local concentration of the drug with minimal systemic toxicity [30].

\subsection{Radioembolization}

It is the delivery of radioactive substances such as Iodine-131 labelled Lipiodol [31] or microspheres containing Yttrium-90 [32]. The injected microspheres will reach the tumor area with selective production of highenergy, low-penetration radiation. Radioembolization carries the advantage of the ability to perform it safely in patients with portal vein thrombosis owing to the minimally embolic effect of $90 \mathrm{Y}$ microspheres [33].

\subsection{Molecular Targeted Therapies}

Many advances changed the management of HCC. Notably, description of molecular and proteomic profiles for HCC prognosis, disease subtyping, and drug selection paved the way for identification of new molecular targeted therapies [34]. Due to the late presence of HCC affected patients, only $15 \%$ can perform curative treatments [35]. In addition, some patients may suffer from 
HCC recurrence post surgical resection. For those patients with advanced or recurrent $\mathrm{HCC}$, systemic therapies can have a role [36].

Pathogenesis of HCC is a multistep complex process. This long process is associated with a large number of epigenetic and genetic alterations that will eventually leads to an alteration in the molecular pathways. These alterations are considered new potential therapeutic targets and include signal transduction pathways, oncogenes and growth factors and their receptors [37]. However, most of the molecular targeted therapies are associated with low tumor response rate. Inhibition of a single signaling pathway may induce feedback activation of other pathways. This may be an important rationale to use combination therapy in clinical trials for a possible beneficial synergistic activity [38].

\section{Sorafenib}

The first successful molecular targeted drug is sorafenib. It is considered the standard systemic therapy for HCC. Sorafenib is an orally administered multikinase inhibitor drug with antiproliferative and antiangiogenic activity [39]. This small molecule multitargeted tyrosine kinase inhibitor (TKI) inhibits vascular endothelial growth factor receptor (VEGFR), platelet-derived growth factor receptor (PDGFR), B-Raf, Fms-related tyrosine kinase (Flt), and c-kit [40,41]. Its main indication is patients with well-preserved liver function (Child-Pugh A class) who have advanced tumors (BCLC C). It is also indicated for tumors progressing on loco-regional therapies [42].

Sorafenib HCC Assessment Randomized Protocol, also known as SHARP trial, is a large randomized phase III study. On the basis of this study, Sorafenib has been approved by the United States (US) Food and Drug Administration (FDA) for the treatment of patients with advanced HCC [43]. This trial succeeded to record an increase in the median overall survival (OS) from 7.9 months in the placebo group to 10.7 months in the sorafenib group. Similarly, a significant benefit of time to progression (TTP) was found with a median of 5.5 months in the sorafenib group and 2.8 months in the placebo group. In turn, the FDA, European Medicine Agency (EMA) and other regulatory authorities in the world have approved sorafenib for advanced HCC treatment [37]. Another large clinical trial was performed for Asian patients with advanced HCC with an overall response rate similar to the SHARP trial [44]. However, the median overall survival was shorter. This difference was mentioned to be related to more advanced disease based on performance status, number of tumor sites, and presence of lung metastases compared with SHARP trial.

An important sorafenib related issue is tolerability and side effects. In the SHARP trial, the most common ad- verse events were diarrhea and hand-foot skin reactions [44]. In a study by Woo et al. [45], 15\% only discontinued the drug due to adverse events. Tolerability was related to Child Pugh class, with significantly longer duration in patients with Child-Pugh class A liver function (233 \pm 240 days) than in those with Child-Pugh class $\mathrm{B}(100 \pm 136$ days; $\mathrm{p}=0.006)$. Even after progression of tumor in some cases (53\% progression rate), those patients who continued treatment with sorafenib or substituted/added concomitant treatment had better survival after progression than patients receiving conservative care only.

An important systemic review of sorafenib studies was performed by Xie and colleagues [46]. Twenty one prospective trials were identified with sorafenib treatment alone or combined with other treatment. Sorafenib succeeded to increase overall survival by $2.3-2.8$ months, extended the time to tumor progression by $1.4-2.7$ months, and increased disease control by $11 \%-19 \%$. Reported major side effects (diarrhea, fatigue, and handfoot syndrome) were evident in less than $15 \%$ of patients, with greater incidence in patients with advanced cirrhosis and those treated with sorafenib in combination with 5-fluorouracil drugs.

In a recent study concerned with effect of combination therapy using sorafenib with cytotoxic chemotherapeutic agents, sorafenib significantly and synergistically enhanced the cytotoxicity of tumor necrosis factor (TNF)related apoptosis inducing ligand (TRAIL) to HCC cells. This effect was found to be derived from sorafenibmediated downregulation of anti-apoptotic proteins [47].

Certain studies looked for a role for sorafenib in cases of HCC recurrence post liver transplantation. Safety profile was a main concern due to combined use of sorafenib with immunosuppressive medications. In a study by Vitale et al. [48], no deterioration of liver graft function occurred and adverse events were manageable with sorafenib dose adjustment. Concerning efficacy, Weinmann et al. [49] reported a median time to progression of 4.1 months and a median overall survival after the initiation of sorafenib treatment of 20.1 months while Vitale et al. [48] reported a median time to progression of 8 months and a median survival from start of therapy of 18 months.

Although no molecular biomarkers are yet available to be recommended to identify the best responders to Sorafenib, studies are ongoing to find predictive biomarkers apart from depending on clinical therapeutic response. A study found that JNK (c-Jun N-terminal kinase) activity was positively correlated with CD133 expression level and inversely correlated with the therapeutic response to Sorafenib. Accordingly, JNK activity may be considered as a new predictive biomarker for response to Sorafenib treatment [50]. 


\subsection{Other Molecular Targeted Therapies}

Due to the limited efficacy of sorafenib, still there is an urgent need to look beyond sorafenib and develop more effective therapy for HCC [51]. In addition, the multiplicity of pathognomonic molecular mechanisms and pathways in hepatocarcinogenesis predicts that dependence on a single-targeted agent might not achieve sustained complete response in HCC. Inhibition of signals at different levels of one of the main pathways or inhibition of two or three different pathways at the same time is more convenient [42]. The different pathways and the corresponding drugs will be presented (Table 2):

VEGF (vascular endothelial growth factor), VEGFR (VEGF receptor), PDGF (platelet-derived growth factor), PDGFR (PDGF receptor), FGR (fibroblast growth factor), FGFR (FGF receptor), EGF (epidermal growth factor) EGFR (EGF receptor), PI3K (Wnt $/ \beta$-catenin, and phosphatidylinositol-3-kinase), PTEN (phosphatase and tensin homologue deleted on chromosome ten), mTOR (Akt/mammalian target of rapamycin), HGF (hepatocyte groth factor), IGF (insulin-like growth factor), IGFR (IGF receptor).

\subsubsection{VEGF/VEGFR, PDGF/PDGFR and FGF/FGFR Targets}

Sunitinib had the chance to reach phase III trials. However, that trial was open label sorafenib controlled randomized study and was prematurely discontinued due to significantly inferior overall survival (7.9 versus 10.2 months) as well as higher rates of major complications [52]. Due to encouraging results of phase II trials for brivanib (median OS of 9.8 months) as well as an acceptable safety profile [53-55], it is more recently

Table 2. Molecular targeted therapies.

\begin{tabular}{|c|c|}
\hline TARGETED PATHWAYS & NAME OF DRUGS \\
\hline $\begin{array}{l}\text { VEGF/VEGFR, } \\
\text { PDGF/PDGFR and } \\
\text { FGF/FGFR }\end{array}$ & $\begin{array}{l}\text { Sorafenib, sunitinib, brivanib, } \\
\text { linifanib (ABT869), vatalinib, } \\
\text { cediranib, dovitinib, axitinib, } \\
\text { pazopanib, regorafinib and } \\
\text { TSU-68, bevacizumab and } \\
\text { ramucirumab }\end{array}$ \\
\hline EGF and EGFR & $\begin{array}{c}\text { Erlotinib, vandetanib, lapatinib, } \\
\text { gefitinib and cetuximab }\end{array}$ \\
\hline PI3K/PTEN/Akt/mTOR & $\begin{array}{l}\text { Perifosine, MK-2206, } \\
\text { sirolimus, evrolimus and } \\
\text { temsirolimus }\end{array}$ \\
\hline HGF/cMET & ARQ 197 and foretinib \\
\hline IGF and IGFR & $\begin{array}{l}\text { OSI 906, cituxumumab } \\
\text { and AVE-1642 }\end{array}$ \\
\hline Epigenetic dysregulation & $\begin{array}{l}\text { Belinostat, verinostat } \\
\text { and romidepsin }\end{array}$ \\
\hline
\end{tabular}

tested in phases III trials in HCC patients, as a first-line blinded to sorafenib (BRISK-FL), as a second-line blinded to placebo (BRISK-PS for unselected population and BRISK-APS for Asian population) and in combination with chemoembolization. Similar to brivanib, linifanib (ABT-869) succeeded to pass a phase II trial with acceptable range of side effects [56] and moved forward to phase III trials as a first line treatment versus sorafenib and as a second line indication. Early studies on dovitinib, another multitargeted TKI, showed good results in inducing significant apoptosis in HCC cells and certainly in sorafenib resistant cells through inhibition of signal transducers and activators of transcription 3 (STAT3). STAT3 showed significant activation in sorafenib resistant cells and so targeting STAT3 may overcome drug resistance in HCC $[57,58]$. As a result, a recent phase II trial started comparing dovitinib as first line therapy versus sorafenib. TSU-68 demonstrated phase I/II potential antitumor activity [59]. In addition, pazopanib, vatalanib, cediranib and axitinib are at early phases of investigations.

Bevacizumab, a recombinant humanized monoclonal antibody, has been used in phase II trials either as single agent [60] or in combination with other molecular targeted therapies and cytotoxic drugs. However, it did not move to phase III trials, may be due to possible severe hemorrhagic events. Ramucirumab is another monoclonal antibody that finished phase II trial [61] and started a phase III trial as a second line therapy after sorafenib failure.

\subsubsection{EGF and EGFR Targets}

A phase II study was accomplished for erlotinib in association with bevacizumab. The overall median survival was 13.7 months [62]. More recently, erlotinib passed to phase III trial in combination with sorafenib. Concerning vandetanib, a phase II trial showed limited clinical activity and the imaging techniques did not detect significant vascular changes after treatment [63]. Lapatinib was investigated in phase II trials but showed suboptimal responses [64,65]. Moreover, the monoclonal antibody cetuximab showed zero objective response rate when was studied in two phase II trials as a single agent $[66,67]$. No better results were obtained when cetuximab was combined with other targeted therapies.

\subsubsection{PI3K/PTEN/Akt/mTOR Targets}

Many listed drugs are still in early phase I/II trials with ongoing patient recruitment. However, everolimus showed a $44 \%$ disease control rate [68] allowing it to pass to phase III trial as a second line treatment after sorafenib failure or intolerance. Temsirolimus is administered intravenously and is in early phases of study for safety profile. 


\subsubsection{HGF/cMET Targets}

Two identified drugs (ARQ 197 and foretinib) are under investigations. ARQ 197 passed phase I trial with reported evidence of efficacy and acceptable safety profile [69]. A phase II trial as a second line treatment is currently running. Foretinib is still in phase I trial.

\subsubsection{IGF and IGFR Targets}

This includes OSI 906, cituxumumab and AVE-1642. They are still under early evaluation as phase I/II trials.

\subsubsection{Epigenetic Dysregulation}

Under clinical trials include belinostat, verinostat and romidepsin. They are in early steps with promising results.

\subsection{Downstaging and Bridging}

The aim of downstaging is to decrease the patient's tumor size and number that do not meet locally acceptable criteria for liver transplantation. A study was performed by $\mathrm{Yu}$ et al. [70] for HCC patients who exceeded the University of California-San Francisco (UCSF) criteria, were downstaged to fit criteria using locoregional therapy and finally underwent liver transplantation. Patients who were successfully downstaged patients before transplantation had excellent tumor-free and overall survival rates, even similar to fit-criteria group.

In 2010, an important international consensus conference was held to implement guidelines for HCC related liver transplantation. Liver transplantation after successful downstaging should achieve a 5-year survival comparable to that of "within criteria" HCC patients. Successful downstaging should include tumor size and number of viable tumors and $\alpha$-fetoprotein concentrations before and after downstaging, then a minimum observation period of 3 months is recommended before considering liver transplantation. Bridging is another strategy that has been developed to treat patients whose HCC is at risk or shows signs of progression while waiting for a graft for 6 months or longer. This strategy is appropriate for patients with UNOS T2 lesions (one nodule $2-5 \mathrm{~cm}$ or three or fewer nodules each $\leq 3 \mathrm{~cm}$ ) [23].

\subsection{Other Investigational Lines of Treatment}

Aiming for better overall survival, decreasing HCC recurrence and better drug delivery to the tumor, many studies were performed while others are still ongoing to try achieving these goals. A recent study tested the in vitro use of gold nanoparticles (GNPs) conjugated with conventional chemotherapy drugs [71]. The cellular proliferation rates in the presence of this combination were statistically lower than those of cells exposed to the chemotherapeutic agents alone. Another study was con- cerned with hepatic cancer stem cells to pave the way for development of new diagnostic, therapeutic and prognostic products that will improve HCC management [72].

In another study performed by Sawada et al. [73], they looked for the development of an anticancer immunotherapy using the carcinoembryonic antigen glypican-3. Generally it was well tolerated with partial response of a patient and stable disease for 2 months for another group of patients after initiation of treatment.

Two systematic reviews were recently performed for the randomized controlled trials performed for adjuvant therapies and adoptive immunotherapies for post operative HCC patients [74,75]. Adoptive immunotherapy was investigated using anti-CD3-activated peripheral blood lymphocytes, cytokine-induced killer cells and lymphokine-activated killer cells. The treatment group with these effector cells could not improve overall survival rate or prevent the multicenteric relapse although they were somewhat able to enhance immunological function and eliminate the small intrahepatic metastases. For adjuvant therapies using, interferon, vitamin K2 analog, acyclic retinoids, transarterial chemotherapies and heparanase inhibitor, results were suboptimal but still promising requiring further studies for some of them. Yet, there is no clear recommendation to use systemic chemotherapy, tamoxifen, immunotherapy, antiandrogen, and herbal drugs for the clinical management of HCC patients [42].

Success of genome-wide association studies (GWAS) and pharmacogenomics raised the possibility of personalized medicine in managing HCC. Three virus-related HCC GWAS were reported for $\mathrm{HCV}$ and $\mathrm{HBV}$ related $\mathrm{HCC}$, each with different locus. This may play a role in future to tailor the best therapeutic targets for HCC patients [76].

\section{Screening and Surveillance}

The aim of screening and surveillance is to early detect HCC and to reduce its disease-related mortality. In China, one randomized controlled trial of surveillance was accomplished comparing twice-yearly ultrasonography and serum $\alpha$-fetoprotein concentration against no surveillance. Survival of screened participants was higher 1, 3 and 5 years when compared to unscreened patients [39]. In addition, Della Corte and Colombo [77] supported screening for liver cancer based on the great differences in response to therapy between screened populations in whom HCC is diagnosed and treated at early stages and patients with more advanced, incidentally detected tumors. However, Cochrane database did not find enough evidence to support or refute the value of alpha-fetoprotein or ultrasound screening, or both, of hepatitis $\mathrm{B}$ surface antigen ( $\mathrm{HBsAg}$ ) positive patients for $\mathrm{HCC}$ and still studies are needed for HCV related HCC [78]. 


\section{Survival and Prognosis}

In general, Child-Pugh class, serum alpha-fetoprotein, tumor size, portal vein thrombosis, and TNM stage are independent prognostic factors for survival among HCC patients. In a recent study, the outcomes in patients with early tumor stage and Child-Pugh class A or B were significantly better with surgical resection than with other treatment modalities and patients who underwent additional transcatheter arterial chemoembolization (as a second-line treatment) after surgical resection had better outcomes than those who underwent surgical resection alone [79].

Moreover, another study found three independent prognostic factors: tumor size, serum indocyanine green retention rate (ICG15) and anatomic wide hepatectomy for better survival post hepatectomy [80]. Gluer et al. [81] additionally found the absence of satellite lesions as a positive predictor factor. For patients who present with extrahepatic involvement or unresectable disease (not suitable or refractory to TACE) and preserved liver condition, systemic therapy is the standard treatment. Prognosis of this group of patients is extremely poor and their median overall survival (OS) is less than 8 months if untreated [51].

\section{REFERENCES}

[1] International Agency for Research on Cancer, 2011. http://www-dep.iarc.fr

[2] R. Masuzaki, H. Yoshida, R. Tateishi, et al., "Staging Systems: Is There a Surgical Staging and a Medical One? Hepatologist's Perspective," Journal of Hepato-BiliaryPancreatic Sciences, Vol. 17, No. 4, 2010, pp. 440-442. doi:10.1007/s00534-009-0240-1

[3] J. N. Vauthey, G. Y. Lauwers, N. F. Esnaola, et al., "Simplified Staging for Hepatocellular Carcinoma," Journal of Clinical Oncology, Vol. 20, No. 6, 2002, pp. 1527-1536. doi:10.1200/JCO.20.6.1527

[4] W. Lu, J. Dong, Z. Huang, et al., "Comparison of Four Current Staging Systems for Chinese Patients with Hepatocellular Carcinoma Undergoing Curative Resection: Okuda, CLIP, TNM and CUPI," Journal of Gastroenterology and Hepatology, Vol. 23, No. 12, 2008, pp. 18741878. doi:10.1111/j.1440-1746.2008.05527.x

[5] K. Okuda, T. Ohtsuki, H. Obata, et al., "Natural History of Hepatocellular Carcinoma and Prognosis in Relation to Treatment. Study of 850 Patients," Cancer, Vol. 56, No. 4, 1985, pp. 918-928. doi:10.1002/1097-0142(19850815)56:4<918::AID-CNCR 2820560437>3.0.CO;2-E

[6] CLIP Investigators, "A New Prognostic System for Hepatocellular Carcinoma: A Retrospective Study of 435 Patients: The Cancer of the Liver Italian Program (CLIP) Investigators," Hepatology, Vol. 28, No. 3, 1998, pp. 751755. doi: $10.1002 /$ hep. 510280322

[7] I. Levy, M. Sherman and Liver Cancer Study Group of the University of Toronto, "Staging of Hepatocellular Carcinoma: Assessment of the CLIP, Okuda, and ChildPugh Staging Systems in a Cohort of 257 Patients in Toronto," Gut, Vol. 50, No. 6, 2002, pp. 881-885. doi:10.1136/gut.50.6.881

[8] C. Y. Hsu, C. Y. Hsia, Y. H. Huang, et al., "Selecting an Optimal Staging System for Hepatocellular Carcinoma: Comparison of 5 Currently Used Prognostic Models," Cancer, Vol. 116, No. 12, 2010, pp. 3006-3014.

doi: $10.1002 /$ cncr. 25044

[9] C. Y. Lin, K. M. Kee, J. H. Wang, et al., "Is the Cancer of the Liver Italian Program System an Adequate Weighting for Survival of Hepatocellular Carcinoma? Evaluation of Intrascore Prognostic Value among 36 Subgroups," Liver International, Vol. 29, No. 1, 2009, pp. 74-81. doi:10.1111/j.1478-3231.2008.01702.x

[10] S. Chevret, J. C. Trinchet, D. Mathieu, et al., "A New Prognostic Classification for Predicting Survival in $\mathrm{Pa}$ tients with Hepatocellular Carcinoma. Groupe d'Etude et de Traitement du Carcinome Hepatocellulaire," Journal of Hepatology, Vol. 31, No. 1, 1999, pp. 133-141. doi:10.1016/S0168-8278(99)80173-1

[11] T. W. Leung, A. M. Tang, B. Zee, et al., "Construction of the Chinese University Prognostic Index for Hepatocellular Carcinoma and Comparison with the TNM Staging System, the Okuda Staging System, and the Cancer of the Liver Italian Program Staging System: A Study Based on 926 Patients," Cancer, Vol. 94, No. 6, 2002, pp. 17601769. doi: $10.1002 / \mathrm{cncr} .10384$

[12] F. D. Huitzil-Melendez, M. Capanu, E. M. O'Reilly, et al., "Advanced Hepatocellular Carcinoma: Which Staging Systems Best Predict Prognosis?" Journal of Clinical Oncology, Vol. 28, No. 17, 2010, pp. 2889-2895. doi:10.1200/JCO.2009.25.9895

[13] M. Kudo, H. Chung and Y. Osaki, "Prognostic Staging System for Hepatocellular Carcinoma (CLIP Score): Its Value and Limitations, and a Proposal for a New Staging System, the Japan Integrated Staging Score (JIS Score)," Journal of Gastroenterology, Vol. 38, No. 3, 2003, pp. 207-215. doi:10.1007/s005350300038

[14] M. Kudo, "Real Practice of Hepatocellular Carcinoma in Japan: Conclusions of the Japan Society of Hepatology 2009 Kobe Congress," Oncology, Vol. 78, No. 1, 2010, pp. 180-188. doi:10.1159/000315740

[15] J. M. Llovet, C. Brú and J. Bruix, "Prognosis of Hepatocellular Carcinoma: The BCLC Staging Classification," Seminars in Liver Disease, Vol. 19, No. 3, 1999, pp. 329338. doi:10.1055/s-2007-1007122

[16] J. Bruix, M. Sherman, Practice Guidelines Committee and American Association for the Study of Liver Diseases, "Management of Hepatocellular Carcinoma," Hepatology, Vol. 42, No. 5, 2005, pp. 1208-1236. doi:10.1002/hep.20933

[17] J. Guy, R. K. Kelley, J. Roberts, et al., "Multidisciplinary Management of Hepatocellular Carcinoma," Clinical Gastroenterology and Hepatology, Vol. 10, No. 4, 2012, pp. 354-362. doi:10.1016/j.cgh.2011.11.008

[18] A. O. Kaseb, Y. M. Abaza and R. E. Roses, "Multidisciplinary Management of Hepatocellular Carcinoma," 
Recent Results in Cancer Research, Vol. 190, 2013, pp. 247-259. doi:10.1007/978-3-642-16037-0 16

[19] R. G. Gish, R. Lencioni, A. M. Di Bisceglie, et al., "Role of the Multidisciplinary Team in the Diagnosis and Treatment of Hepatocellular Carcinoma," Expert Review of Gastroenterology \& Hepatology, Vol. 6, No. 2, 2012, pp. 173-185. doi:10.1586/egh.11.105

[20] V. Mazzaferro, S. Bhoori, C. Sposito, et al., "Milan Criteria in Liver Transplantation for HCC: An EvidenceBased Analysis on 15 Years of Experience," Liver Transplantation, Vol. 17, No. 2, 2011, pp. S44-S57. doi:10.1002/lt.22365

[21] V. Mazzaferro, E. Regalia, R. Doci, et al., "Liver Transplantation for the Treatment of Small Hepatocellular Carcinomas in Patients with Cirrhosis," The New England Journal of Medicine, Vol. 334, No. 11, 1996, pp. 693-699. doi:10.1056/NEJM199603143341104

[22] F. Y. Yao, L. Xiao, N. M. Bass, et al., "Liver Transplantation for Hepatocellular Carcinoma: Validation of the UCSF-Expanded Criteria Based on Preoperative Imaging," American Journal of Transplantation, Vol. 7, No. 11, 2007, pp. 2587-2596. doi:10.1111/j.1600-6143.2007.01965.x

[23] P. A. Clavien, M. Lesurtel, P. M. Bossuyt, et al., "Recommendations for Liver Transplantation for Hepatocellular Carcinoma: An International Consensus Conference Report," The Lancet Oncology, Vol. 13, No. 1, 2012, pp. e11e22. doi:10.1016/S1470-2045(11)70175-9

[24] S. Padma, J. B. Martinie and D. A. Iannitti, "Liver Tumor Ablation: Percutaneous and Open Approaches," Journal of Surgical Oncology, Vol. 100, No. 8, 2009, pp. 619-634. doi: $10.1002 /$ jso. 21364

[25] R. Lencioni, "Loco-Regional Treatment of Hepatocellular Carcinoma," Hepatology, Vol. 52, No. 2, 2010, pp. 762773. doi:10.1002/hep. 23725

[26] R. Lencioni, C. Bartolozzi, D. Caramella, et al., "Treatment of Small Hepatocellular Carcinoma with Percutaneous Ethanol Injection. Analysis of Prognostic Factors in 105 Western Patients," Cancer, Vol. 76, No. 10, 1995, pp. $1737-1746$. doi:10.1002/1097-0142(19951115)76:10<1737::AID-CN CR2820761010>3.0.CO;2-P

[27] F. Brunello, A. Veltri, P. Carucci, et al., "Radiofrequency Ablation versus Ethanol Injection for Early Hepatocellular Carcinoma: A Randomized Controlled Trial," Scandinavian Journal of Gastroenterology, Vol. 43, No. 6, 2008, pp. 727-735. doi:10.1080/00365520701885481

[28] K. Takayasu, S. Arii, I. Ikai, et al., "Prospective Cohort Study of Transarterial Chemoembolization for Unresectable Hepatocellular Carcinoma in 8510 Patients," $G a$ stroenterology, Vol. 131, No. 2, 2006, pp. 461-469. doi:10.1053/j.gastro.2006.05.021

[29] S. N. Goldberg, C. J. Grassi, J. F. Cardella, Society of Interventional Radiology Technology Assessment Committee and the International Working Group on ImageGuided Tumor Ablation, "Image Guided Tumor Ablation: Standardization of Terminology and Reporting Criteria," Journal of Vascular and Interventional Radiology, Vol. 20, No. 7, 2009, pp. S377-S390. doi:10.1016/j.jvir.2009.04.011

[30] M. Varela, M. I. Real, M. Burrel, et al., "Chemoembolization of Hepatocellular Carcinoma with Drug Eluting Beads: Efficacy and Doxorubicin Pharmacokinetics," Journal of Hepatology, Vol. 46, No. 3, 2007, pp. 474-481. doi:10.1016/j.jhep.2006.10.020

[31] J. L. Raoul, D. Guyader, J. F. Bretagne, et al., "Prospective Randomized Trial of Chemoembolization versus Intraarterial Injection of 131I-Labeled-Iodized Oil in the Treatment of Hepatocellular Carcinoma," Hepatology, Vol. 26, No. 5, 1997, pp. 1156-1161.

[32] R. Salem, R. J. Lewandowski, M. F. Mulcahy, et al., "Radioembolization for Hepatocellular Carcinoma Using Yttrium-90 Microspheres: A Comprehensive Report of LongTerm Outcomes," Gastroenterology, Vol. 138, No. 1, 2010, pp. 52-64. doi:10.1053/j.gastro.2009.09.006

[33] L. M. Kulik, B. I. Carr, M. F. Mulcahy, et al., "Safety and Efficacy of 90Y Radiotherapy for Hepatocellular Carcinoma with and without Portal Vein Thrombosis," Hepatology, Vol. 47, No. 1, 2008, pp. 71-81. doi:10.1002/hep. 21980

[34] B. I. Carr, "Some New Approaches to the Management of Hepatocellular Carcinoma," Seminars in Oncology, Vol. 39, No. 4, 2012, pp. 369-373. doi:10.1053/j.seminoncol.2012.05.007

[35] R. S. Finn, "Development of Molecularly Targeted Therapies in Hepatocellular Carcinoma: Where Do We Go Now?" Clinical Cancer Research, Vol. 16, No. 2, 2010, pp. 390-397. doi:10.1158/1078-0432.CCR-09-2084

[36] C. Frenette and R. Gish, "Targeted Systemic Therapies for Hepatocellular Carcinoma: Clinical Perspectives, Challenges and Implications," World Journal of Gastroenterology, Vol. 18, No. 6, 2012, pp. 498-506. doi:10.3748/wig.v18.i6.498

[37] M. Cervello, J. A. McCubrey, A. Cusimano, et al., "Targeted Therapy for Hepatocellular Carcinoma: Novel Agents on the Horizon," Oncotarget, Vol. 3, No. 3, 2012 pp. 236-260.

[38] K. E. O'Reilly, F. Rojo, Q. B. She, et al., "mTOR Inhibition Induces Upstream Receptor Tyrosine Kinase Signaling and Activates Akt," Cancer Research, Vol. 66, No. 3, 2006, pp. 1500-1508. doi:10.1158/0008-5472.CAN-05-2925

[39] A. Forner, J. M. Llovet and J. Bruix, "Hepatocellular Carcinoma," Lancet, Vol. 379, No. 9822, 2012, pp. 12451255. doi:10.1016/S0140-6736(11)61347-0

[40] S. M. Wilhelm, C. Carter, L. Tang, et al., "BAY 43-9006 Exhibits Broad Spectrum Oral Antitumor Activity and Targets the RAF/MEK/ERK Pathway and Receptor Tyrosine Kinases Involved in Tumor Progression and Angiogenesis," Cancer Research, Vol. 64, No. 19, 2004, pp. 7099-7109. doi:10.1158/0008-5472.CAN-04-1443

[41] Y. S. Chang, J. Adnane, P. A. Trail, et al., "Sorafenib (BAY 43-9006) Inhibits Tumor Growth and Vascularization and Induces Tumor Apoptosis and Hypoxia in RCC Xenograft Models," Cancer Chemotherapy and Pharmacology, Vol. 59, No. 5, 2007, pp. 561-574. doi:10.1007/s00280-006-0393-4

[42] European Association for the Study of the Liver and 
European Organisation for Research and Treatment of Cancer, "EASL-EORTC Clinical Practice Guidelines: Management of Hepatocellular Carcinoma," Journal of Hepatology, Vol. 56, No. 4, 2012, pp. 908-943.

[43] J. M. Llovet, S. Ricci, V. Mazzaferro, et al., "SHARP Investigators Study Group. Sorafenib in Advanced Hepatocellular Carcinoma," The New England Journal of Medicine, Vol. 359, No. 4, 2008, pp. 378-390. doi:10.1056/NEJMoa0708857

[44] A. L. Cheng, Y. K. Kang, Z. Chen, et al., "Efficacy and Safety of Sorafenib in Patients in the Asia-Pacific Region with Advanced Hepatocellular Carcinoma: A Phase III Randomised, Double-Blind, Placebo-Controlled Trial," The Lancet Oncology, Vol. 10, No. 1, 2009, pp. 25-34. doi:10.1016/S1470-2045(08)70285-7

[45] H. Y. Woo, J. Heo, K. T. Yoon, et al., "Clinical Course of Sorafenib Treatment in Patients with Hepatocellular Carcinoma," Scandinavian Journal of Gastroenterology, Vol. 47, No. 7, 2012, pp. 809-819. doi: $10.3109 / 00365521.2012 .683040$

[46] B. Xie, D. H. Wang and S. J. Spechler, "Sorafenib for Treatment of Hepatocellular Carcinoma: A Systematic Review," Digestive Diseases and Sciences, Vol. 57, No. 5, 2012, pp. 1122-1129. doi:10.1007/s10620-012-2136-1

[47] K. Nojiri, K. Sugimoto, K. Shiraki, et al., "Sorafenib and TRAIL Have Synergistic Effect on Hepatocellular Carcinoma," International Journal of Oncology, Vol. 42, No. 1, 2013, pp. 101-108.

[48] A. Vitale, G. Lombardi, R. Ramirez Morales, et al., "Potential Impact of Sorafenib on the Survival Benefit of Liver Transplantation for Hepatocellular Carcinoma," Digestive and Liver Disease, Vol. 44, No. 5, 2012, pp. 361-362. doi:10.1016/j.dld.2012.02.014

[49] A. Weinmann, I. M. Niederle, S. Koch, et al., "Sorafenib for Recurrence of Hepatocellular Carcinoma after Liver Transplantation," Digestive and Liver Disease, Vol. 44, No. 5, 2012, pp. 432-437. doi:10.1016/j.dld.2011.12.009

[50] S. Hagiwara, M. Kudo, T. Nagai, et al., "Activation of JNK and High Expression Level of CD133 Predict a Poor Response to Sorafenib in Hepatocellular Carcinoma," British Journal of Cancer, Vol. 106, No. 12, 2012, pp. $1997-$ 2003. doi:10.1038/bjc.2012.145

[51] S. L. Chan, T. Mok and B. B. Ma, "Management of Hepatocellular Carcinoma: Beyond Sorafenib," Current Oncology Reports, Vol. 14, No. 3, 2012, pp. 257-266. doi:10.1007/s11912-012-0233-0

[52] A. Cheng, Y. Kang, D. Lin, et al., "Phase III Trial of Sunitinib (Su) versus Sorafenib (So) in Advanced Hepatocellular Carcinoma (HCC)," Journal of Clinical Oncology, Vol. 29, No. 15, 2011, p. 4000.

[53] R. S. Finn, Y. K. Kang, M. Mulcahy, et al., "Phase II, Open-Label Study of Brivanib as Second-Line Therapy in Patients with Advanced Hepatocellular Carcinoma," Clinical Cancer Research, Vol. 18, No. 7, 2012, pp. 20902098. doi:10.1158/1078-0432.CCR-11-1991

[54] J. A. Garcia and L. R. Roberts, "Phase II, Open-Label Study of Brivanib as First-Line Therapy in Patients with Advanced Hepatocellular Carcinoma," Journal of Hepa- tology, Vol. 56, No. 2, 2012, pp. 486-487. doi:10.1016/j.jhep.2011.07.033

[55] J. W. Park, R. S. Finn, J. S. Kim, et al., "Phase II, OpenLabel Study of Brivanib as First-Line Therapy in Patients with Advanced Hepatocellular Carcinoma," Clinical Cancer Research, Vol. 17, No. 7, 2011, pp. 1973-1983. doi:10.1158/1078-0432.CCR-10-2011

[56] H. C. Toh, P. J. Chen, B. I. Carr, et al., "Phase 2 Trial of Linifanib (ABT-869) in Patients with Unresectable or Metastatic Hepatocellular Carcinoma," Cancer, Vol. 119, No. 2, 2012, pp. 380-387. doi:10.1002/cncr.27758

[57] W. T. Tai, A. L. Cheng, C. W. Shiau, et al., "Dovitinib Induces Apoptosis and Overcomes Sorafenib Resistance in Hepatocellular Carcinoma through SHP-1-Mediated Inhibition of STAT3," Molecular Cancer Therapeutics, Vol. 11, No. 2, 2012, pp. 452-463. doi:10.1158/1535-7163.MCT-11-0412

[58] K. F. Chen, H. L. Chen, C. Y. Liu, et al., "Dovitinib Sensitizes Hepatocellular Carcinoma Cells to TRAIL and Tigatuzumab, a Novel Anti-DR5 Antibody, through SHP-1Dependent Inhibition of STAT3," Biochemical Pharmacology, Vol. 83, No. 6, 2012, pp. 769-777. doi:10.1016/j.bcp.2011.12.035

[59] F. Kanai, H. Yoshida, R. Tateishi, et al., "A Phase I/II Trial of the Oral Antiangiogenic Agent TSU-68 in Patients with Advanced Hepatocellular Carcinoma," Cancer Chemotherapy and Pharmacology, Vol. 67, No. 2, 2011, pp. 315-324. doi:10.1007/s00280-010-1320-2

[60] A. B. Siegel, E. I. Cohen, A. Ocean, et al., "Phase II Trial Evaluating the Clinical and Biologic Effects of Bevacizumab in Unresectable Hepatocellular Carcinoma," Journal of Clinical Oncology, Vol. 26, No. 18, 2008, pp. $2992-$ 2998. doi:10.1200/JCO.2007.15.9947

[61] A. X. Zhu, R. S. Finn, M. F. Mulcahy, et al., "A Phase II Study of Ramucirumab as First-Line Monotherapy in Patients (pts) with Advanced Hepatocellular Carcinoma (HCC)," Journal of Clinical Oncology, Vol. 28, 2010.

[62] A. O. Kaseb, E. Garrett-Mayer, J. S. Morris, et al., "Efficacy of Bevacizumab plus Erlotinib for Advanced Hepatocellular Carcinoma and Predictors of Outcome: Final Results of a Phase II Trial," Oncology, Vol. 82, No. 2, 2012, pp. 67-74. doi:10.1159/000335963

[63] C. Hsu, T. S. Yang, T. I. Huo, et al., "Vandetanib in Patients with Inoperable Hepatocellular Carcinoma: A Phase II, Randomized, Double-Blind, Placebo-Controlled Study," Journal of Hepatology, Vol. 56, No. 5, 2012, pp. 1097-1103. doi:10.1016/j.jhep.2011.12.013

[64] R. K. Ramanathan, C. P. Belani, D. A. Singh, et al., "A Phase II Study of Lapatinib in Patients with Advanced Biliary Tree and Hepatocellular Cancer," Cancer Chemotherapy and Pharmacology, Vol. 64, No. 4, 2009, pp. 777-783. doi:10.1007/s00280-009-0927-7

[65] T. Bekaii-Saab, J. Markowitz, N. Prescott, et al., "A Multi-Institutional Phase II Study of the Efficacy and tolerability of Lapatinib in Patients with Advanced Hepatocellular Carcinomas," Clinical Cancer Research, Vol. 15, No. 18, 2009, pp. 5895-5901. doi:10.1158/1078-0432.CCR-09-0465

[66] A. X. Zhu, K. Stuart, L. S. Blaszkowsky, et al., "Phase 2 
Study of Cetuximab in Patients with Advanced Hepatocellular Carcinoma," Cancer, Vol. 110, No. 3, 2007, pp. 581-589. doi:10.1002/cncr.22829

[67] Y. Gruenwald, L. Wilkens, M. Gebel, et al., "A Phase II Open-Label Study of Cetuximab in Unresectable Hepatocellular Carcinoma: Final Results," ASCO Annual Meeting Proceedings, Vol. 25, No. 18S, 2007, p. 4598.

[68] A. X. Zhu, T. A. Abrams, R. Miksad, et al., "Phase 1/2 Study of Everolimus in Advanced Hepatocellular Carcinoma," Cancer, Vol. 117, No. 22, 2011, pp. 5094-5102. doi:10.1002/cncr.26165

[69] P. Zucali, A. Santoro, C. Rodriguez-Lope, et al., "Final Results from ARQ 197-114: A Phase Ib Safety Trial Evaluating ARQ 197 in Cirrhotic Patients (pts) with Hepatocellular Carcinoma (HCC)," Journal of Clinical Oncology, Vol. 28, 2010, Abs 4137.

[70] C. Y. Yu, H. Y. Ou, T. L. Huang, et al., "Hepatocellular Carcinoma Downstaging in Liver Transplantation," Transplantation Proceedings, Vol. 44, No. 2, 2012, pp. 412414. doi:10.1016/j.transproceed.2012.01.043

[71] C. Tomuleasa, O. Soritau, A. Orza, et al., "Gold Nanoparticles Conjugated with Cisplatin/ Doxorubicin/Capecitabine Lower the Chemoresistance of Hepatocellular Carcinoma-Derived Cancer Cells," Journal of Gastrointestinal and Liver Diseases, Vol. 21, No. 2, 2012, pp. 187196.

[72] J. Ji and X. W. Wang, "Clinical Implications of Cancer Stem Cell Biology in Hepatocellular Carcinoma," Seminars in Oncolog, Vol. 39, No. 4, 2012, pp. 461-472. doi:10.1053/j.seminoncol.2012.05.011

[73] Y. Sawada, T. Yoshikawa, D. Nobuoka, et al., "Phase I Trial of a Glypican-3-Derived Peptide Vaccine for Advanced Hepatocellular Carcinoma: Immunologic Evidence and Potential for Improving Overall Survival," Clinical Cancer Research, Vol. 18, No. 13, 2012, pp. 36863896. doi:10.1158/1078-0432.CCR-11-3044

[74] J. H. Zhong, L. Ma, L. C. Wu, et al., "Adoptive Immu- notherapy for Postoperative Hepatocellular Carcinoma: A Systematic Review," International Journal of Clinical Practic, Vol. 66, No. 1, 2012, pp. 21-27. doi:10.1111/j.1742-1241.2011.02814.x

[75] J. H. Zhong, H. Li, L. Q. Li, et al., "Adjuvant Therapy Options Following Curative Treatment of Hepatocellular Carcinoma: A Systematic Review of Randomized Trials,' European Journal of Surgical Oncology, Vol. 38, No. 4, 2012, pp. 286-295. doi:10.1016/j.ejso.2012.01.006

[76] D. Miki, H. Ochi, C. N. Hayes, et al., "Hepatocellular Carcinoma: Towards Personalized Medicine," Cancer Science, Vol. 103, No. 5, 2012, pp. 846-850. doi:10.1111/j.1349-7006.2012.02242.x

[77] C. Della Corte and M. Colombo, "Surveillance for Hepatocellular Carcinoma," Seminars in Oncology, Vol. 39, No. 4, 2012, pp. 384-398. doi:10.1053/j.seminoncol.2012.05.002

[78] R. Aghoram, P. Cai and J. A. Dickinson, "Alpha-Foetoprotein and/or Liver Ultrasonography for Screening of Hepatocellular Carcinoma in Patients with Chronic Hepatitis B," Cochrane Database of Systematic Reviews, Vol. 9, 2012, Article ID: CD002799.

[79] S. S. Lee, H. S. Shin, H. J. Kim, et al., "Analysis of Prognostic Factors and 5-Year Survival Rate in Patients with Hepatocellular Carcinoma: A Single-Center Experience," Korean Journal of Hepatology, Vol. 18, No. 1, 2012, pp. 48-55. doi:10.3350/kjhep.2012.18.1.48

[80] T. Ochiai, H. Ikoma, Y. Murayama, et al., "Factors Resulting in 5-Year Disease-Free Survival after Resection of Hepatocellular Carcinoma," Anticancer Research, Vol. 32, No. 4, 2012, pp. 1417-1422.

[81] A. M. Gluer, N. Cocco, J. M. Laurence, et al., "Systematic Review of Actual 10-Year Survival Following Resection for Hepatocellular Carcinoma," HPB (Oxford), Vol. 14, No. 5, 2012, pp. 285-290. doi:10.1111/j.1477-2574.2012.00446.x 